\title{
Microperforate (pinhole) hymen and infertility: A rare case report
}

\author{
Davut Güven $^{1}$, Kadir Bakay ${ }^{2 *}$, Serkan Kuruoglu ${ }^{1}$ \\ ${ }^{1}$ Department of Obstetrics and Gynecology, 19 Mayis University School of Medicine, Samsun, Turkey \\ ${ }^{2}$ Private Hospital Department of Obstetrics and Gynecology, Turkey \\ Email: drkadirbakay@gmail.com
}

Received 15 May 2012; revised 19 June 2012; accepted 30 June 2012

\begin{abstract}
Introduction: Female genital tract anomalies may have a devastating impact on the potential for sexual activity and fertility. Microperforate (pinhole) hymen is one of them. Microperforate hymen can lead to infertility, primary amenorrhea, acute or chronic pelvic pain, abnormal vaginal bleeding, dysuria, pollakiuria or a foul-smelling vaginal discharge. Aim: We aim to report a patient who had intercourse problems and infertility complaints later found to have a microperforate hymen. Discussion: Pinhole opening of hymen may permit passage of fluids. Consequently spontaneous pregnancy can occur rarely. A few reports showed pregnancies with intact hymen without penetration of penis. But in general patients have fertility problems. Conclusion: The results of the surgery are similar in all techniques and the particular surgical centre will have its own preference of which technique it prefers since after diagnosis definitive treatment is surgical and effective. Failure to manage these patients correctly may have long-term sequelae for their psychological, sexual and reproductive health.
\end{abstract}

Keywords: Hymen; Pinhole; Microperforate; Infertility

\section{INTRODUCTION}

Female genital tract anomalies are widely variable. And these anomalies may have a devastating impact on the potential for sexual activity and fertility. Varieties of the hymen are one of them. Hymenal anomalies are derived from incomplete degeneration of the central portion of the hymen. These anatomic variants include imperforate, pinhole (microperforate), septate, and cribriform. Pinhole hymen is a congenital obstructive vaginal membrane with a minimal opening and incomplete obstructive pathology usually permitting normal menstrual flow. This is a rare form and frequently mistaken for an imperforate

${ }^{*}$ Corresponding author. hymen. The opening up pinhead can be a passage for menstrual bleeding and sperms. Therefore these women may have delayed diagnosis.

\section{AIM}

We aim to report a patient who had intercourse problems and infertility complaints later found to have a microperforate hymen. Even though this is a unique case we aim to highlight the importance of vaginal examination and definitive treatment that can have a dramatic effect on sexual health and fertility.

\section{CASE REPORT}

A 29 years old woman presented to our clinic complaining of infertility. She was married for 1.5 years and stated that she had regular menstruations, periods lasting about five days each month. There were no urinary symptoms, dysmenorrhea nor spotting. She also mentioned pain and difficulty with attempted or completed intercourse. She was diagnosed with vaginismus and infertility several times, but vaginal examination was not possible because the patient did not want it.

On examination she appeared to be normal. Secondary sex characters were well developed. Visual inspection of her external genitalia revealed a thick and tight membrane covering the vagina with only a pinpoint opening (Figure 1). We described that as pinhole (microperforated) hymen. And transabdominal sonography revealed normal uterus and adnexa, and no other abnormal findings were noted. Hematological and biochemical test results were normal as well.

Excess hymenal tissue was resected to create a functional hymenal ring under general anesthesia. An electrocautery was used to excise hymenal tissue. Then redundant fragment of the membrane was cut away to allow adequate coitus. To prevent introital stenosis, we sutured the edges to the introitus. Examination after the resection showed a normal vagina and cervix. She had intercourse after two mounts of the operation. 


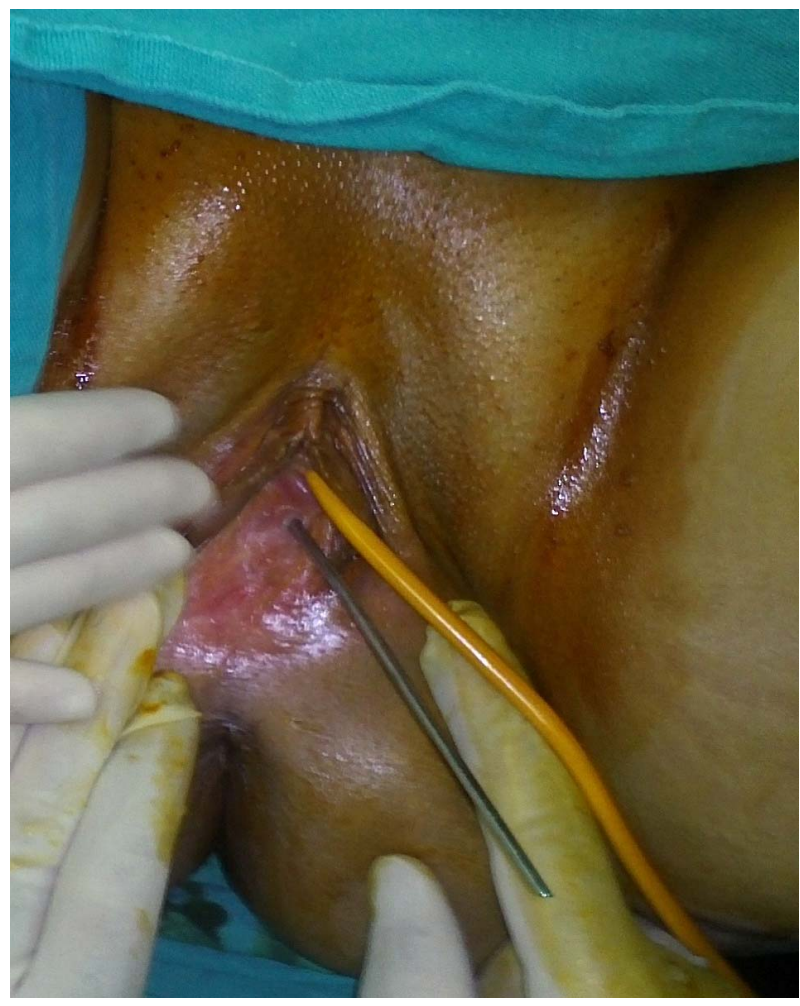

Figure 1. Pinhole (microperforate) hymen.

\section{DISCUSSION}

Hymen is an embryologic remnant that usually perforates during the later stages of embryonic development and remains as a thin fold of mucous membrane. Hymenal anomalies occur if the genital tubercule fails to breakdown or rupture $[1,2]$.

These anomalies may cause many symptoms. Common symptoms seen in hymenal malformations include primary amenorrhea, acute or chronic pelvic pain, abnormal vaginal bleeding, dysuria, pollakiuria or a foulsmelling vaginal discharge. Non-obstructive anomalies like microperforate, septate or cribriform hymen may even be found incidentally on routine examination.

Sometimes patients may present with vaginismus and these patients may not permit even an external vaginal examination. Usually their main complaint is dispauronia and unsuccessful intercourse due to a closed vaginal orifice. Our patient was evaluated for infertility for six months and because she did not want a vaginal examination diagnosis was delayed.

Pinhole opening of hymen may permit passage of fluids. Consequently spontaneous pregnancy can occur rarely. A few reports showed pregnancies with intact hymen without penetration of penis [3,4]. But in general patients present with fertility problems.

In rare cases microperforate hymen can be found in the secondary closure of the hymen. Two stations can cause the secondary closure of the hymen; following sexual child abuse and another two cases in pregnancy; one followed by a previous surgical incision of the hymen at an early age and the other closed spontaneously during pregnancy [5].

\section{CONCLUSIONS}

Microperforate hymen can lead to recurrent urinary tract infections and ascending pelvic infections. Recurrent urinary tract infections can occur because of pooling of urine in the functional urogenital sinus and invasion into the urethra [6]. So pelvic examination is a must in prepubertal and adolescent patients who have complaints of recurrent urinary infections.

Failure to manage these patients correctly may have long-term sequelae for their psychological, sexual and reproductive health [2].

The results of the surgery are similar in all techniques and the particular surgical centre will have its own preference of which technique it prefers since after diagnosis definitive treatment is surgical and effective [2].

\section{REFERENCES}

[1] Bhatnagar, K.P., Nettleton, G.S. and Nakajima, S.T. (2002) Female genital anomalies affecting reproduction. Fertility and Sterility, 78, 899-915.

[2] Edmonds, D.K. (2003) Congenital malformations of the genital tract and their management. Best Practice \& Research Clinical Obstetrics \& Gynaecology, 17, 19-40.

[3] Goto, K., Yoshinari, H., Tajima, K. and Kotsuji, F. (2006) Microperforate hymen in a primigravida in active labor: A case report. The Journal of Reproductive Medicine, 51, 584-586.

[4] Topcuoglu, M.A., Koc, O., Timuroglu, T. and Donmez, M. (2010) Microperforate hymen and pregnancy: A case report. http://tipdergisi.ibu.edu.tr/arsiv/2010/123/9.pdf

[5] Onan, M.A., Turp, A.B., Taskiran, C., et al. (2005) Spontaneous closure of the hymen during pregnancy. American Journal of Obstetrics and Gynecology, 193, 889-891. doi:10.1016/j.ajog.2005.03.025

[6] Bakos, O. and Berglund, L. (1999) Imperforate hymen and ruptured hematosalpinx: A case report with a review of the literature. Journal of Adolescent Health, 24, 226228. doi:10.1016/S1054-139X(98)00077-9 